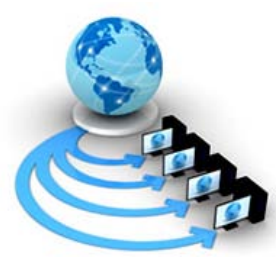

Volume 9, No. 2, March-April 2018

\title{
INFLUENCE OF ANGER AND ANXIETY IN ELEVATING THE BLOOD PRESSURE: USING MACHINE LEARNING APPROACHES
}

\author{
Satyanarayana Nimmala \\ Dept. of CSE, CVRCE \\ Hyderabad, India
}

\author{
Dr. Y. Ramadevi \\ Dept. of CSE, CBIT \\ Hyderabad, India
}

\author{
R.Sahith \\ Dept. of CSE, CVRCE \\ Hyderabad, India
}

\begin{abstract}
In the present days most of the people are suffering from anger and anxiety at some point in time in their life. Though getting anger is common for many but frequently getting anger, continuing the same for a longer period of time, has an adverse effect on the human body. Suffering from Anxiety may enable the fight or flight mode of the central nervous system. This elevates the blood pressure for a shorter period of time. There may be different reasons for a person to suffer from anger and anxiety, but if it is chronic it may elevate blood pressure of a person. If the elevated blood pressure is more than the normal range, it is considered as high blood pressure (HBP) or hypertension. In this paper, we considered anger level and anxiety level of a person to predict whether the person is prone to HBP or not. Anger and anxiety level of a person is measured using response obtained from the set of predefined questionnaire. We considered 1000 records For Experimental analysis, where each record consists of anger and anxiety level of a person along with systolic, diastolic and mean arterial blood pressure. Experiments are conducted using various machine learning algorithms. We used $50 \%$ records to train the model, $50 \%$ records to test the model. Results unfold that there is a significant influence of anger level and anxiety level in elevating the blood pressure.
\end{abstract}

Keywords: Hypertension, Anger, Anxiety, Machine learning, Data Mining.

\section{INTRODUCTION}

Blood Pressure (BP) is represented as Systolic over Diastolic blood pressure. If systolic blood pressure exceeds $140 \mathrm{mmHg}$ or diastolic blood pressure exceeds $90 \mathrm{mmHg}$ on repeated measurements then it is treated as HBP [1]. Now a day's HBP is one of the leading cause of heart attack and stroke. It may not be a serious problem if it is diagnosed and treated earlier, but undiagnosed HBP may cause a serious health problem. There are many reasons which may elevate the BP like unhealthy diet, lack of physical exercise, excess bad cholesterol, obesity [2][9], age, anger, anxiety etc. But in this paper, we focused on the impact of anger, and anxiety in elevating the BP. Data is collected from a group of 1000 people aged between 20 and 65 . Their systolic and diastolic blood pressures recorded using U80B digital blood pressure Monitor. High Blood pressure (HBP) mainly affected by the Cardiac Output (CO) and Total Peripheral Resistance (TPR). Mathematically, it can be written as [3][10].

$$
\mathrm{BP}=\mathrm{CO} * \mathrm{TPR}
$$

Here, CO is affected by increased venous return or Stroke volume or Heart Rate or Sympathetic activity. Whereas TPR is affected by the resistance that acts against the blood flow in the arteries [4]. The arteries may show resistance to blood flow because of, a blood clot in blood vessels or presence of fat inside the blood vessels or damaged blood vessels.
CO affects the Systolic blood pressure, whereas TPR affects the Diastolic Blood Pressure.

$$
\begin{aligned}
& \mathrm{BP}=\text { Systolic/ Diastolic } \\
& \text { Systolic } / \text { Diastolic = CO } / \mathrm{TPR}
\end{aligned}
$$

Sometimes, we may consider Mean Arterial Pressure which can be calculated as

$$
\text { MAP }=(2 * \text { Diastolic }+ \text { Systolic }) / 3
$$

If MAP is within the range then all organs, tissues will get enough blood, oxygen, and nutrients [5].

Stress, anger, and anxiety may not spike blood pressure for the longer duration of time [6] but uncontrolled anger may affect relationships, career, mental and physical health. In this paper, we considered anger and anxiety levels for experimental analysis. Anger and anxiety levels are measured using the response of an individual for the set of predefined questions. For anger measurement, we set 10 predefined questions and for anxiety measurement, we set 20 predefined questions. 1000 people are interviewed and their response is noted on a scale of 0 to 3 . The mean value of the response, for all the questions on anger and anxiety, are used in the prediction process along with age. Table I represents the level of hypertension. 
Table I. Blood Pressure Range

\begin{tabular}{|c|c|c|c|c|}
\hline BP & Low & Normal & Borderline & High \\
\hline Systolic & $<90$ & $90-130$ & $131-140$ & $>140$ \\
\hline Diastolic & $<60$ & $60-80$ & $81-90$ & $>90$ \\
\hline
\end{tabular}

\section{IMPACT OF ANGER AND ANXIETY}

Blood pressure of a person is elevated because of biological and psychological changes. Biological changes are like age, increase in obesity level and total blood cholesterol. Psychological changes are such as anger, anxiety, stress, depression, and fear. But the exact influence of each of these factors is left for research. In this paper, we considered anger and anxiety levels of a person to predict whether a person is prone HBP or not.

\section{A. Anger Measurement}

Anger may be the result of impatience, frustration, irritation and many others. It may be positive emotion at some time but most of the time it is not good for health and state of mind. The way how anger is handled has a significant effect on heart and mind. Frequent explosive anger may lead to serious consequences like elevated blood pressure, the rise in a heartbeat and pulse rate. When a person gets angry then the fight or flight mode of Sympathetic Nervous System gets activated. As a response nerves send more blood to muscles and brain, which elevates the blood pressure [7]. Though suppressing and ignoring the anger is not good for health but letting it go is also not good. So everyone should mastery over anger in a way that the impact of it to be as minimal as possible. The literature says anger and blood pressure may not associate for a longer period of time[8]. But our experimental results show there is a significant effect of anger along with the anxiety of a person in elevating the blood pressure. Our proposed technique measures the level of anger by using the responses obtained from the predefined questionnaire. Sample questions used for anger measurement are like waiting for anything annoys me, gets angry for the delay in completion of any assignment, gets angry if things won't go on my path, and I find difficult to forgive people who did wrong to me. We used 10 such questions and for each question, the answer is marked as one of the following option a) no, never b) yes, rarely c) yes, often d) yes, most of the time. In the data preprocessing phase option, a is considered as 0 , option b is considered as 1 , option $\mathrm{c}$ is considered as 2 and option $d$ is considered as 3 . Based on the mean value of all the answers we considered either floor value or ceil value of mean for experimental analysis.

\section{B. Anxiety Measurement}

Stress and anxiety are slightly different, even they represented on the same scale. The active form of stress may consider as anxiety, and the active form anxiety may consider as depression. Now a day's stress is one of the key factors that impact the quality of our regular life. If stress is chronic, that happening frequently, it may become anxious. Anxiety response creates specific thought pattern in mind, which gets executed repeatedly. The person, who is a victim of anxiety, thinks again and again about the worst possible outcome of an ambiguous situation, where the possibility of happening best is more. Factors such as negative thinking, fear, insecurity, lack of something compared to others, thinking about a specific thing that may happen in future, lack of confidence, doing wrong things which are not ethical, not getting things right as per his or her perception, expectations from friends, relatives, and closed ones etc., may be the triggering factors for anxiety. When a person is anxious, fight or flight mode of Sympathetic Nervous System gets activated [9]. As said earlier it elevates the blood pressure. However, Anxiety and long-term HBP may not be linked [10]. The body produces a surge of hormones such as adrenaline and cortical when we are in the anxious situation. This, in turn, may tighten the arteries [11][12]. Our experimental analysis also reveals that there is a significant impact of anxiety, in raising the blood pressure. Our proposed technique measures the level of anxiety by using the responses obtained from the predefined questionnaire. Sample questions used for anxiety measurement are like, the existence of constant fear about something, facing breathing difficulty often, feeling of not having desired things in life, often scared without the clear reason, often aware of the heart beat without doing physical exercise, and sense of dryness in the mouth. We used 20 such questions and for each question, the answer is marked as one of the following option a) no, never b) yes, rarely c) yes, often d) yes, most of the time. In the data preprocessing phase option, a is considered as 0 , option b is considered as 1 , option c is considered as 2 and option $\mathrm{d}$ is considered as 3 . Based on the mean value of all the answers we considered either floor value or ceil value of mean for experimental analysis.

\section{Proposed Methodology}

In this paper, we used a data mining classification technique. Classification is the technique used to predict the class label of a data record or to represent a descriptive analysis of data record for taking effective decisions [13[14]. It is also called as a supervised approach. It uses mathematics, statistics, probability distributions and artificial intelligence. The classification model consists of two stages: In stage 1 . Training stage, where the model is trained by a set of records, whose class labels are already known. In stage 2. Testing stage, where the model is going to predict class labels of a set of records whose class labels are unknown, also called as test records. There are various classifiers, but we have used classifiers supported by the WEKA for experimental analysis. 
Table II. Details of Attributes.

\begin{tabular}{|c|c|c|c|c|c|c|}
\hline Attribute Number & Attribute & Minimum & Maximum & Mean & Standard Deviation & Attribute Type \\
\hline 2 & Anger level & 0 & 3 & 1.797 & 0.618 & Numeric \\
\hline 3 & Anxiety level & 0 & 3 & 1.139 & 0.664 & Numeric \\
\hline
\end{tabular}

Table III. Class wise Accuracy Details.

\begin{tabular}{|c|c|c|c|c|c|c|}
\hline Classifier Name & Class & TP Rate & FP Rate & Precision & Recall & F-Measure \\
\hline \multirow{2}{*}{ NAÏVE BAYES } & YES & 0.300 & 0.161 & 0.533 & 0.300 & 0.384 \\
\hline & NO & 0.839 & 0.700 & 0.662 & 0.839 & 0.740 \\
\hline \multirow{2}{*}{$\mathrm{J} 48$} & YES & 0.100 & 0.000 & 1.000 & 0.100 & 0.182 \\
\hline & NO & 0.834 & 0.143 & 0.909 & 0.834 & 0.870 \\
\hline \multirow{2}{*}{ REP TREE } & YES & 0.102 & 0.000 & 1.000 & 0.102 & 0.185 \\
\hline & NO & 1.000 & 0.898 & 0.657 & 1.000 & 0.793 \\
\hline \multirow{2}{*}{$\begin{array}{c}\text { RANDOM } \\
\text { FOREST }\end{array}$} & YES & 0.109 & 0.000 & 1.000 & 0.109 & 0.196 \\
\hline & NO & 1.000 & 0.891 & 0.658 & 1.000 & 0.794 \\
\hline
\end{tabular}

The above and below measures are used to measure the performance of the classifier, these are standard measures used.

Table IV. Comparative Analysis of Accuracy and Error rates of Different algorithms

\begin{tabular}{|c|c|c|c|c|c|}
\hline S. Number & Algorithm & Accuracy \% & MAE & RMSE & RAE \% \\
\hline 2 & NaiveBayes Theorem & 63.4 & 0.4396 & 0.4723 & 92.421 \\
\hline 3 & J48 Algorithm & 65.8 & 0.4439 & 0.4696 & 93.3218 \\
\hline 4 & REP TREE & 67 & 0.4346 & 0.4643 & 91.5933 \\
\hline 5 & Random Forest & 67.1429 & 0.4333 & 0.4627 & 91.4498 \\
\hline
\end{tabular}

MAE: Mean absolute error, RMSE: Root mean squared error, RAE: Relative absolute error

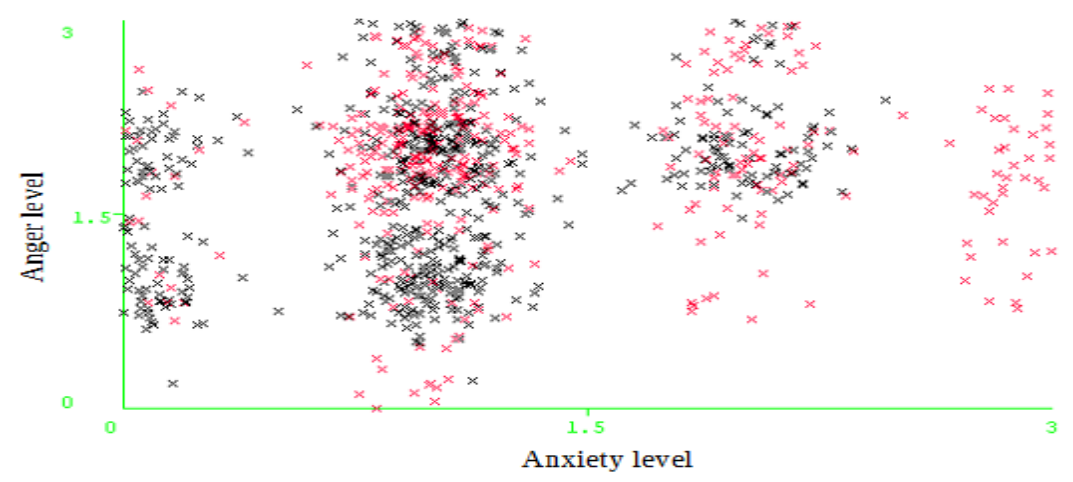

Figure. 1. Blood pressure prediction using Anxiety and Anger

\section{EXPERIMENTAL RESULTS AND ANALYSIS}

We collected data from 1000 people. Each record in the data set has anger level, anxiety level, systolic blood pressure, diastolic blood pressure, and mean arterial pressure. Where mean arterial blood pressure and TABLE1 are used to find the class label attribute. If anger level and anxiety level are below 1 we considered their floor value and more than one we considered their ceil value for the better prediction. The classwise accuracy details of different classifiers are shown in Table III. Figure. 1 represents anxiety level on $\mathrm{X}$-axis, where the minimum age is 0 , maximum age is 3 . $\mathrm{Y}$-axis represents anger level, where minimum anger level is 0 , and maximum anger level is 3. Here red ' $x$ ' represents data records which are predicted as YES, black ' $\mathrm{x}$ ' represents data records which are predicted as NO. Here Table IV represents accuracy and error rates different classifiers.

\section{CONCLUSION AND FUTURE WORK}

In this paper, anger, and anxiety levels of a person to predict whether a person is prone to HBP or not. From the experimental analysis we obtained, if the anger, anxiety levels are greater than or equal to two, then the person is most likely to become a victim of high blood pressure. Results unfold; the influence of anxiety is more than the anger in elevating the blood pressure. The experiment also reveals that if a person anger level less than one and but anxiety level is more than 2 , then the person is more likely to become a victim of HBP. In future, we would like to consider few more attributes like the age of a person, total blood cholesterol, Obesity, smoking and 
drinking levels of a person to predict high blood pressure more accurately. These may improve the performance of the classifier also.

\section{REFERENCES}

[1] A. Alwan, "Global status report on noncommunicable diseases 2010,” World Health Organization, 2011.

[2] L. Hendricks, S. Bore and D. Aslinia, "The Effects of Anger on the Brain and Body," in National forum journal of counseling and addiction, vol. 2, pp. 1-12, 2013.

[3] E.E. Gillis and J.C. Sullivan, "Sex Differences in Hypertension Recent Advances, " in Recent Advances in Hypertension, vol.68, pp.1322- -1327, 2016.

[4] WHO: World Health Organization [---------Online-------] Available:http://www.who.int/gho/ncd/risk_factors/blood_p ressure_prevalence_text/en/

[5] N.Satyanarayana, A.Avinash and Dr. Y.Ramadevi--, "Predicting High Blood Pressure using Age and Obesity," in International Journal of Computer and Mathematical Sciences, vol.6, pp.27--30, 2017.

[6] P.Pandey, E.Kyung Lee and D.Pompili, “A Distributed Computing Framework for Real-Time Detection of Stress And of Its Propagation in a Team," in IEEE Journal of Biomedical and health Informatic, vol.20, pp.1502--1512, 2016.

[7] S.C.Millasseau, R.P. Kelly, J.M. Ritter and P.J. Chowienzyk, "The vascular impact Of Aging and vasoactive Drugs: Comparison of two digital volume pulses Measurements," in American journal of Hypertension Sciences, vol.16, pp.467--472, 2003.

[8] N.Satyanarayana, CH. Ramalinga swamy , Y.Ramadevi, "Survey of Classication Techniques in Data Mining," in International Journal of Innovative Science, Engineering and Technology, vol.1, pp.268--279, 2014.

[9] J.K.K.Vishram, A.Borglykke, A.H.Andreasen, J.Jeppesen And H.Ibsen, "Impact of Age on the Importance of Systolic and Diastolic Blood Pressures for Stroke Risk," in Hypertension, vol.60, pp.1117--1123, 2012.

[10] N Richard, “ Obesity Related Hypertension, " in The Ochner Journal, vol.9, pp.133--136, 2009.

[11] J. O. Serna, W. Van Moer and K.Barbé “ Using alternating Kalman filtering to analyze oscillometric blood pressure Waveforms," in IEEE Transactions on Instrumentation and Measurement, vol.62, pp.2621-2628, 2013.

[12] M.Ursino, C.Cristalli, “ A mathematical study of some Biomechanical factors affecting the oscillometric blood Pressure measurement," in IEEE Transactions on Biomedical Engineering, vol.43, pp.761--768, 1996.

[13] M.Forouzanfar, "Ratio-Independent Blood Pressure Estimation by Modeling the Oscillometric Waveform Envelope," in IEEE Transactions on Instrumentation and Measurement, vol.63, pp.2501--2503, 2014.

[14] C.F. Babbs, "Oscillometric measurement of systolic and Diastolic blood pressures validated in a physiologic Mathematical model," in Biomedical Engineering Online, vol.11, pp.1--22, 2012. 